\title{
Les valeurs et les actes : une perspective transdisciplinaire pour l'éducation à l'environnement
}

Sylvie Kergreis

\section{OpenEdition}

Journals

Édition électronique

URL : http://journals.openedition.org/ere/2147

DOI : $10.4000 /$ ere. 2147

ISSN : 2561-2271

Éditeur

Centr'ERE

\section{Référence électronique}

Sylvie Kergreis, "Les valeurs et les actes : une perspective transdisciplinaire pour l'éducation à l'environnement », Éducation relative à l'environnement [En ligne], Volume 8 | 2009, mis en ligne le 20 décembre 2009, consulté le 21 février 2020. URL : http://journals.openedition.org/ere/2147 ; DOI : 10.4000/ere.2147 


\title{
Les valeurs et les actes : une perspective transdisciplinaire pour l'éducation à l'environnement
}

\author{
Sylvie Kergreis
}

1 «Choisir des valeurs et les mettre en œuvre » est un projet de vie que chacun s'efforce de tenir, un projet que nous souhaitons proposer à nos enfants ou aux jeunes en formation. Mais qu'en est-il de nos infortunes, de nos ratés et de nos imperfections? Comment les expliquons-nous? En termes éducatifs, quels repères donnons-nous à ce sujet? L'éducation à l'environnement est particulièrement propice à l'exploration du lien existant entre nos valeurs, nos actes concrets et les forces diverses des dynamiques écologiques et sociales qui nous entourent. Cerner les contraintes qui s'imposent aux volontés individuelles peut aider à préciser les potentialités offertes à chacun. En adoptant une perspective transdisciplinaire (Morin, 1997), il est possible d'envisager les valeurs invoquées dans l'éthique comme une facette particulière d'une notion plus générale, l'expression multiforme de l'énergie qui traverse les corps vivants et leurs milieux de vie, et se trouve régulée dans le fonctionnement des systèmes environnementaux et sociaux. En effet, toutes les disciplines, qu'elles soient scientifiques, artistiques, religieuses ou philosophiques abordent cette notion de valeur, de pulsion, qui rythme la vie et le positionnement de chaque élément imbriqué dans différents systèmes. Travailler sur l'articulation, la transformation et la régulation de cette énergie / valeur au sein des éco-socio-systèmes offre des perspectives intéressantes pour mieux comprendre ce qui se joue dans nos choix personnels, dans nos décisions collectives ou dans nos actes concrets vis-à-vis de l'environnement. Les deux études présentées ici, qui s'appuient sur la problématique du bocage en Bretagne, apportent des exemples de cette déclinaison des valeurs, à plusieurs niveaux de systèmes imbriqués. Elles permettent de proposer des pistes de réflexion et d'action pour l'éducation à l'environnement, aussi bien par la méthodologie choisie que par les résultats obtenus. 


\section{Réalisme modeste et positionnement scientifique}

2 Pour ne pas se perdre dans la circularité des systèmes complexes, dans l'idéologie des discours ou dans l'opacité de nos propres motivations, la recherche présentée s'appuie sur trois partis pris.

3 Le « réalisme modeste » (Dewitte, 2001) incite à tenir compte de la matérialité et de la finitude des organismes et des systèmes, pris dans leur mouvement perpétuel de transformation. Cette attention au "déjà-là " vaut aussi bien pour le monde environnemental et social que pour les constructions intellectuelles. Une position modeste ne s'exempte pas de références au réel ou aux théories passées: elle les relativise à travers les idéologies ou les théories scientifiques, en les resituant dans leurs contextes. Un équilibre est recherché entre matérialisme, constructivisme, apports des sciences dites « positivistes » et approches plus qualitatives ou intuitives.

4 Les poly, inter et trans disciplinarités permettent ces allers et retours féconds entre raison et intuition. Construire une approche transdisciplinaire nécessite un travail collectif. Il faut partager une bonne connaissance des théories et pratiques de plusieurs disciplines applicables à l'objet étudié (poly). L'interdisciplinarité construit de nouvelles approches par articulation progressive entre les démarches de ces disciplines (inter), non sans heurts entre visions du monde. Puis un travail de synthèse et d'intuition permet l'émergence de nouvelles conceptions et explications, dans les interstices des apports précédents et au-delà des contradictions rencontrées (trans). Pour Edgar Morin (1997), le travail sur la complexité des systèmes brouille souvent les repères et autorise, au final, tous les discours. Seules la rigueur et la précision dans la construction de cette pensée nouvelle peuvent nous garder en éveil, avec leurs exigences de questionnement, de modélisation, de réfutation / validation d'hypothèses et de constants retours sur les expériences vécues et la réalité observée.

5 Selon Legay (1997), dans ce type de démarche systémique où les multiples angles d'attaque peuvent modifier les jugements, aucune position dite « objective » n'est plus tenable. Le chercheur, en décidant de choix épistémologiques, tranche parmi des incertitudes, comme tout un chacun. Il faut donc préciser préalablement le point de vue d'où part la recherche, l'objectif personnel ou social poursuivi, parfois de manière implicite. Cette exigence inscrit le chercheur dans le monde et non hors de lui, ce qui permet d'éviter une description externe de la circularité, souvent propice à la confusion des discours.

\section{Les valeurs, dans la circularité de la vie}

6 De l'infime décision humaine à l'extase mystique, du choix des techniques à l'organisation du travail, de l'échange économique aux décisions collectives, de la mélodie musicale aux jeux de lumière dans la forêt, du geste patient du jardinier à la circulation mondiale des biens et services, de la puissance des végétaux à l'engagement de toute une vie, du grondement des vagues au sourire fugace... l'énergie vitale se faufile et s'exprime dans la matérialité environnementale, humaine et sociale, dans les actions, les discours et les rêves. Tout comme les systèmes écologiques, les systèmes humains et sociaux imbriqués régulent cette énergie bio-physico-chimique et psychique, pour leur propre organisation et survie. Ce lien énergie-valeur, perçu 
intuitivement, est travaillé par plusieurs disciplines, dont l'économie (GeorgescuRoegen, 1979) ou la psychologie (Feertchak, 1996 ; Peeters, 1999). Une circularité relie les évaluations infimes et générales, dans un processus mêlant énergie, valeurs et idéologies qui régule l'ensemble des productions culturelles aussi bien que les actions individuelles, les corps (Mauss, 1936), les sciences et les techniques (Habermas, 1990) ou les échanges économiques (Passet, 1974). Ces évaluations sont le miroir de nos sociétés et de leurs rapports nature-culture (Descola, 2005).

7 Au niveau culturel général, les valeurs donnent sens à la vie individuelle et collective, orientent les actions, inspirent les lois, justifient les normes sociales tout comme les éthiques individuelles ou les codes moraux. Ces matrices forment les structures de régulation de l'univers des relations humaines, mais elles en sont également le produit. Elles expriment la résultante des forces diverses qui naissent des multiples évaluations suscitées par les activités et les rencontres des êtres humains entre eux et avec leur environnement, à plusieurs niveaux imbriqués.

8 Au niveau écologique, l'énergie physique, chimique ou biologique s'exprime à travers le développement spontané de la flore et de la faune, les manifestations du climat, etc. Les activités humaines tout comme les techniques et les outils, organisés socialement, s'articulent à ces processus énergétiques. Le travail physique des corps, l'énergie psychique, les plaisirs et les souffrances ressenties constituent les données primaires de la régulation qui s'instaure entre nos motivations et la puissance vitale qui nous entoure.

9 Au niveau individuel, cette énergie s'exprime sous forme de pulsions, sensations, émotions, affects, désirs, motivations. Les intérêts, les normes et les valeurs viennent compléter ce mélange d'évaluations, plus ou moins implicites ou explicites, qui dirigent l'organisme humain et la personne dans ses activités diverses. Ces évaluations se mêlent intimement aux connaissances et aux représentations du monde, progressivement construites à travers les histoires de chacun et les appartenances sociales.

$10 \mathrm{Au}$ niveau inter-individuel, la rencontre des motivations personnelles crée des possibilités de conflit ou de coopération. Prendre le pouvoir sur l'autre, être dominé, construire un rapport de réciprocité, s'entraider, donner, recevoir, etc., toutes les relations humaines gèrent des échanges d'énergie à travers des règles, des normes, des valeurs ou des contraintes physiques (l'esclavage et l'exploitation sont des moyens trouvés par certains pour s'exempter du travail nécessaire à la survie...).

11 Au niveau du groupe social, les discussions, les arrangements, les coutumes et les normes permettent de réguler ces rapports interpersonnels par des évaluations partagées (ou subies) concernant les actions, les objets et les personnes, en fonction des situations, selon plusieurs registres de jugement possible (Boltansky et Thevenot, 1991). Les conflits, les négociations et les accords rythment la vie collective, dans des structures sociales plus ou moins complexes, introduisant là aussi pouvoir, oppression ou coopération, structurant nos rapports à l'environnement.

12 Au niveau institutionnel, les règles et les lois permettent de trancher entre les intérêts contradictoires des groupes et régulent la société. Les valeurs y sont invoquées comme grands principes. Ce niveau étatique (ou européen, par exemple) organise aussi la gestion concrète du bien commun: les voies de circulation, la protection contre les 
risques, l'accumulation et la diffusion des connaissances, etc. sont autant de services, souvent oubliés, qui médiatisent notre rapport au monde naturel.

Ainsi, l'ensemble du système se boucle et évolue, généralement lentement, mais parfois aussi à travers des crises violentes ou des effondrements.

\section{Les valeurs environnementales et leurs conséquences concrètes}

La prise de conscience des problèmes environnementaux a favorisé l'expression de nouveaux principes ou finalités orientant les choix sociaux. Plusieurs travaux de psychologues ont tenté de comprendre comment les "valeurs environnementales" s'inscrivaient dans le panel des grandes valeurs humaines. Rejoignant les analyses de l'éthique environnementale, ce principe de préservation à long terme de l'environnement a été différencié à travers des motivations " égoïstes ", "altruistes » ou «biocentrés » (Stern et Dietz, 1994). En utilisant le modèle des valeurs universelles de Schwartz $(1992,2006)$ (voir la figure 1) ${ }^{1}$ et en opposant les aspects biocentrés aux aspects anthropocentrés (égoïstes et altruistes), Schultz et Zelezny (1999) montrent que les valeurs dites biocentrées (préservation de la nature pour elle-même) sont uniquement corrélées positivement avec celles de «l'universalisme», tandis qu'elles sont corrélées négativement avec le « pouvoir » et la « tradition ».

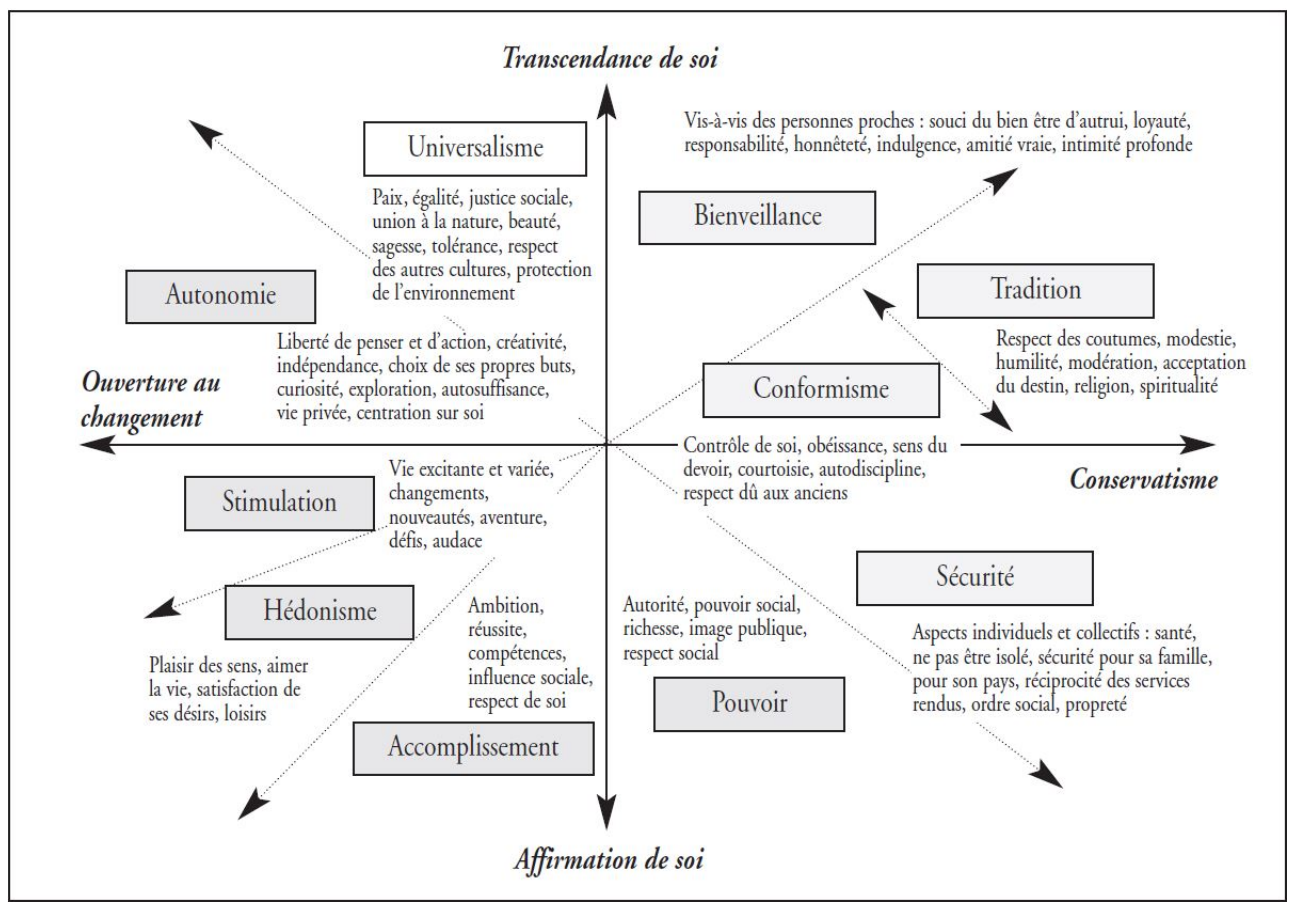

Figure 1 : Une présentation des valeurs de Schwartz $(1992,2006)$

«L'hédonisme » et les valeurs «d'ouverture " n'ont pas de liens particuliers. Les valeurs environnementales anthropocentrées (préservation de la nature pour soi ou pour les humains en général) sont corrélées positivement au " pouvoir» et aux trois valeurs du «conservatisme» (tradition, sécurité, conformité) et négativement à la «bienveillance » (qui concerne le souci des autres proches). 

l'environnement peine à être pris en compte en tant que tel: les enjeux sociaux ou personnels prennent le plus souvent le dessus au cours des décisions collectives ou individuelles (Lafaye et Thévenot, 1993; Billaud, 1996). Ainsi, les valeurs environnementales, en tant que grands principes posant la préservation de l'environnement comme finalité, ne sont pas autonomes : elles sont liées à certaines valeurs sociales et disparaissent souvent à leur profit. Leurs définitions par les êtres humains en font des constructions sociales, mais plus encore, c'est leur mise en œuvre qui pose problème.

La définition des états « objectifs » de l'environnement et des bonnes conditions de sa préservation pose la question de l'existence de connaissances descriptives exemptes d'évaluations humaines ou sociales (Beauvois, 1994). Les sciences et l'expertise deviennent lieu de débat et de remises en cause (Theys, 2000). Les êtres humains se battent et négocient pour définir «leur réalité ». L'environnement est un terme flou qui contient aussi bien la biodiversité que le paysage, eux-mêmes sujets à diverses définitions et évaluations. Cependant, la nature n'est pas toujours muette. La prolifération des algues vertes sur les plages ou les coulées de boues emportant des maisons en sont des manifestations concrètes. La plasticité des discours se heurte alors à une réalité externe, résistante voire insaisissable. Schultz et Zelezny (1999) s'interrogent sur le lien entre les valeurs et les actes en remarquant que même les valeurs anthropocentrées ne conduisent pas systématiquement à des comportements pro-environnementaux. Pour eux, la construction d'échelles d'attitudes proenvironnementales censées prédire de tels comportements à partir d'opinions générales se révèle décevante. D'autres travaux sur les attitudes arrivent aux mêmes conclusions : l'observation des habitudes in situ est souvent plus prédictive des actions futures que le recueil de discours. (Channouf et al., 1996). Ainsi, pour comprendre comment peuvent se concrétiser d'éventuelles «valeurs environnementales », il faut s'intéresser de très près aux situations concrètes, pour saisir comment le sens donné à l'intention se trouve modifié par diverses évaluations jusqu'à rencontrer l'énergie physique du système qui nous entoure. L'étude des techniques et des pratiques concrètes permet d'explorer les relations homme-nature sous un autre angle (Jollivet, 1992).

\section{Construction de l'étude}

18 Notre recherche s'est intéressée à la problématique sociale de la disparition du bocage en Bretagne, qui mêle des aspects sociaux et environnementaux. Au-delà des haies, auxquelles sont principalement sensibles les habitants locaux, des scientifiques ${ }^{2}$ en sont venus à étudier le réseau des "bordures de champ " (haies, fossés, talus, bandes d'herbe ou bosquets) pour comprendre l'articulation entre les aspects environnementaux et sociaux de cet objet complexe. Ces espaces perçus comme naturels sont en réalité tributaires des techniques employées par les agriculteurs pour les entretenir. En effet, historiquement, ce sont eux qui co-habitent quotidiennement avec ces lisières, où les dynamiques écologiques rencontrent les organisations sociales, dans une profonde interaction mutuelle (Kergreis, 2007).

Dans notre étude, le "déjà-là » concerne les vieilles haies héritées de modes de vie passés, avec leurs émondages particuliers construits dans des rapports sociaux 
spécifiques (les troncs pour les propriétaires / les branches pour les fermiers, par exemple). Il concerne les connaissances pratiques des agriculteurs, celles des techniciens organisant la replantation de nouvelles haies, aussi bien que les connaissances scientifiques accumulées sur la question.

Notre réflexion transdisciplinaire s'est construite à travers les apports d'écologues, agronomes et hydrologues du Laboratoire de l'INRA SAD (Institut national de la recherche agronomique, Sciences pour l'Action et le Développement) Paysage, Rennes ainsi que plusieurs travaux menés dans un cadre universitaire ${ }^{3}$, en sociologie, économie de la régulation, anthropologie et psychologie. Le positionnement de la recherche ${ }^{4}$ est centré sur "la protection de l'environnement", entendue comme une conjonction d'actions concrètes, bio-physico-chimiques, permettant de gérer, améliorer ou conserver la qualité de l'eau, des sols et de la biodiversité. Les chercheurs de l'INRA SAD Paysage nous ont aidés à préciser les conditions de cette protection, relativement aux haies et bordures de champ. Parallèlement à ce choix assumé de nous appuyer sur leur expertise (souvent prudente, voire réticente), l'analyse du débat social a donné l'occasion de resituer leurs propositions et valeurs dans l'ensemble des argumentaires (Kergreis, 2001).

21 À cause de ce positionnement, nous avons été particulièrement vigilants pour établir, au niveau des valeurs invoquées, un gradient démêlant les finalités environnementales des finalités sociales. Nous parlerons de «valeurs environnementales» en ce qui concerne la biodiversité, l'eau ou le sol, tandis que nous inclurons dans les «valeurs sociales » le paysage ou les plaisirs pris dans la nature. Ces valeurs seront articulées avec des attitudes qui, dans notre choix de définition, concernent des actions ou des objets précis. Dans l'étude 1, nous analysons un choix technique individuel, en illustrant le lien valeurs - attitude - comportement - contraintes physiques. Dans l'étude 2, nous cherchons à mesurer l'évolution des points de vue au cours de choix collectif portant sur des aménagements ruraux. Nous observons cette évolution sur des valeurs, des connaissances concrètes et des arrangements sociaux, dans différents contextes. Les intérêts sociaux et économiques tout comme les lois et règlements, souvent passés sous silence dans les modèles psychologiques, sont insérés dans notre analyse.

\section{De l'intention à l'action}

Notre première étude (Kergreis et Thenail, 2008) s'est intéressée aux attitudes et comportements des agriculteurs en Bretagne, concernant l'entretien du bas de leurs bordures de champs. Cent treize agriculteurs ${ }^{5}$ ont répondu à un questionnaire fermé, construit à partir de douze entretiens semi-directifs. Le questionnaire reprenait certaines phrases exprimées par les 12 agriculteurs initialement rencontrés pour en faire des questions d'opinion, en demandant le degré d'accord de chacun, sur une échelle de 1 à 7. Les réponses étant ainsi quantifiées, une analyse de régression a permis de sélectionner les aspects ayant les plus forts liens avec l'intention d'action (voir la figure 2). 


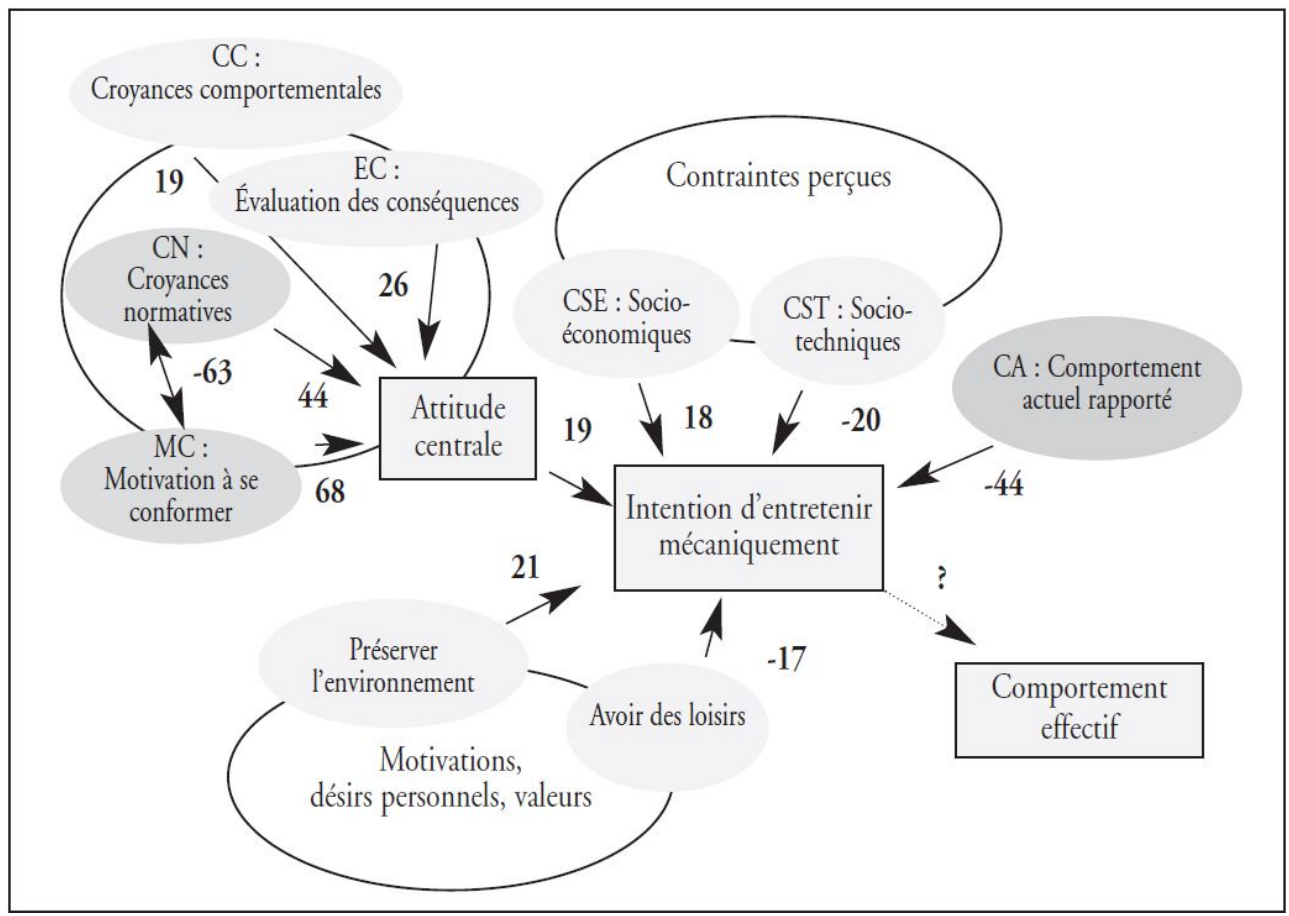

Figure 2 : «Entretenir mécaniquement le bas des bordures de champ » relatives à un objet ou à comportement projeté. Nous avons choisi un modèle théorique (Azjen et Fishbein, 1980) qui préconise de cibler un comportement précis pour améliorer les prédictions des résultats obtenus. Nous nous sommes centrés sur une pratique pro-environnementale : l'entretien des fossés ou bords herbeux avec des outils mécaniques (plutôt que des herbicides chimiques). Dans le modèle utilisé, l'attitude générale est en lien avec des croyances évaluatives, quant aux conséquences de l'action (CC et $\mathrm{EC})^{6}$ ou aux normes sociales (CN et $\mathrm{MC}$ ). Elle se combine avec les contraintes perçues (CSE et CST), autres croyances concernant la possibilité d'émettre le comportement selon le contexte. D'autres auteurs ont introduit d'autres facteurs. Nous avons retenu les motivations, basées sur des désirs personnels ou sur de grandes valeurs donnant sens à la vie, ainsi que le comportement passé ou actuel (CA) vis-à-vis de l'objet (Perugini et Bagozzi, 2001 ; Ajzen, 2002).

Dans nos résultats, les habitudes d'action semblent avoir le poids le plus important. Les agriculteurs utilisant déjà $d u$ désherbant ne semblent pas souhaiter revenir à l'entretien mécanique. L'attitude générale, partagée par la majorité des agriculteurs interrogés, considère qu'il faut entretenir, sinon je vais être envahi ${ }^{7}$. Au chapitre des désirs ou valeurs personnelles invoquées, avoir des loisirs comme tout le monde entre en contradiction avec l'entretien mécanique, car ce dernier demande d'intervenir plus souvent que l'entretien chimique. Au contraire, certains agriculteurs désirent protéger l'environnement, ce qui va dans le sens de l'action visée.

Les aspects normatifs s'expriment à travers une évaluation de la situation sociale. Certains agriculteurs refusent l'entretien mécanique en arguant du fait que si la commune et les particuliers ne respectent pas l'environnement, je ne vois pas pourquoi moi je le ferais (MC). D'autres activent des normes construites dans leur voisinage rural ou par les agriculteurs entre eux $(\mathrm{CN})$ : on est jugé par rapport à l'entretien que l'on fait; les 
agriculteurs pensent qu'il est indispensable d'entretenir. Certaines normes sociales peuvent être si anciennes et si bien intériorisées qu'elles apparaissent comme une évaluation personnelle :j'aime bien que ce soit propre, pour moi, pour mon plaisir personnel (EC). Au côté des valeurs personnelles et des aspects normatifs, des connaissances concrètes apparaissent, en lien avec le comportement projeté. Certaines pensées peuvent être contradictoires, illustrant ainsi les débats internes de chacun (CC): l'utilisation de désherbant total a plusieurs types d'inconvénients (effondrement des talus, apparition de mauvaises herbes résistantes) mais l'entretien mécanique des bords de champ coûte plus cher que l'entretien chimique. Ce dilemme, partagé par beaucoup, fonctionne comme un conflit mental, au moment du choix d'action. Des contraintes externes sont perçues par l'individu, qu'elles soient liées à sa situation socio-économique ou au milieu naturel : je voudrais bien faire de l'entretien mécanique mais je ne dispose pas de temps suffisant ou cela me coûte trop cher (CSE) ou bien il y a des endroits où c'est impossible (CST).

Cet exemple concret permet de cerner toute la complexité et l'ambivalence d'un choix d'action. Le jeu des désirs personnels, des grandes valeurs, des normes sociales s'articule avec les croyances et les perceptions liées aux situations. Nous voyons également ici l'importance des habitudes, le rôle des contraintes de temps, de travail ou d'argent ou celui des contraintes physiques du terrain. Ainsi, malgré ses valeurs personnelles, un même agriculteur peut être amené à agir de manière contradictoire, pour plusieurs raisons : de la plus occasionnelle et concrète (par exemple, passer du désherbant quand il est pressé) à la plus spécifique et sociale (par exemple, faire attention à un bord de fossé bien visible de la route). L'incertitude ou l'ambivalence des choix s'expliquent par ces nombreux facteurs.

\section{Du rôle des valeurs dans les discussions}

Notre deuxième étude (Kergreis, 2004) s'est intéressée au rôle et au devenir des valeurs dans une discussion collective, toujours dans un objectif pro-environnemental. Trois concertations locales déjà existantes, concernant des aménagements fonciers et ruraux, ont été suivies et analysées pendant trois ans, en Bretagne. Les représentants des agriculteurs, de collectivités locales et d'associations environnementales se réunissaient en (A) pour mener une action concrète locale, en (B) pour construire des projets à long terme, en $(\mathrm{C})$ pour résoudre un conflit départemental. Le contexte général présentait une forte remise en cause de l'agriculture productiviste, accusée de polluer l'eau potable et de détruire les milieux naturels. Les participants ont été interrogés de manière semi-directive à la suite de l'observation des concertations.

Dans ces trois cas concrets, l'impact environnemental des accords a été analysé sur le terrain, en lien avec le laboratoire d'écologie et d'agronomie. Parallèlement, des minirencontres d'étudiants ont été organisées, dans des lycées de l'ouest de la France, entre des classes préparant à des brevets de technicien supérieur (1er cycle d'enseignement supérieur), les unes à spécialisation agricole, les autres à spécialisation environnementale ${ }^{8}$. Il s'agissait d'étudier l'impact de différents types de discussions sur les accords trouvés et sur l'évolution des points de vue des participants. Un questionnaire, proposé avant et après la décision collective, abordait les points de vue sur des questions fermées, à l'aide d'une échelle en sept points, ce qui permettait de mesurer statistiquement l'évolution des opinions. Ce questionnaire avait été construit en quatre parties: descriptifs de bordures de champ, valeurs associées au choix des 
bordures (pour protéger l'eau, pour ne pas gêner l'agriculture, par exemple), techniques employées pour les entretenir, arrangements sociaux pour se répartir les avantages et les coûts relatifs à l'existence et l'entretien des bordures (droit de propriété, contrat, obligations légales, hausse des prix agricoles, achat des bordures, etc.). Les discussions demandaient de choisir des types de bordures dans un espace rural, selon diverses modalités (choix ouvert sur huit types de photos (D1), ou huit descriptions de bordure (D2), ou huit valeurs recherchées (D3)... choix contraint activant la nouvelle norme agri-environnementale (D4). Nos hypothèses générales prévoyaient que la diffusion des nouvelles valeurs environnementales présentait un contexte favorable à l'évolution des accords et des points de vue dans ce sens, grâce au phénomène de la polarisation et de l'influence minoritaire dans les groupes de discussion, mis en évidence par certaines études de psychologie sociale (Paicheler, 1976 ; Moscovici et Doise, 1992).

Les résultats obtenus ont montré, au contraire, que les valeurs et les positions agricoles productivistes sortaient renforcées des concertations observées et des débats entre étudiants, malgré le contexte général et la présence d'agriculteurs (ou d'étudiants agricoles) minoritaires pro-environnementaux dans certains groupes. Ce résultat est d'autant plus intéressant que la "protection de l'eau » est apparue comme une valeur consensuelle, une finalité générale de choix d'action, reconnue par tous, au cours des discussions et des accords verbaux observés, tout comme dans les opinions personnelles mesurées. Ainsi, cet accord manifesté publiquement et validé personnellement par chacun des participants masque une réalité sociale plus complexe, où les accords finaux, l'évolution des points de vue et les réalisations concrètes ${ }^{9}$ n'assurent pas la mise en œuvre de la « valeur environnementale » partagée.

Les évolutions des points de vue des étudiants au cours de la discussion illustrent la complexité du phénomène. Pour les quatre types de connaissances proposées par le questionnaire, huit phrases différenciaient des choix possibles selon des positions plutôt "environnementalistes", plutôt "productivistes agricoles", ou plutôt "agriculture durable» (terrain d'entente possible au cours des discussions). Contrairement à nos hypothèses, les points de vue des étudiants en environnement ont évolué positivement ${ }^{10}$ sur des aspects agricoles productivistes (degrés d'accord supérieurs concernant les valeurs, les techniques ou les bordures de ce type), tandis que les étudiants en agriculture ont le plus souvent maintenu leurs positions. Les points de vue des étudiants en agriculture pro-environnementaux (agriculture durable) ont parfois même évolué vers des postures plus conformes à la majorité de leur groupe professionnel (agriculture productiviste), ce que nous avons également observé dans une concertation réelle.

31 Nos résultats montrent que tous les niveaux d'évaluation (valeurs, lois, normes, intérêts socio-économiques, désirs personnels) sont activés dans la discussion et la décision collective. Au moment de la décision, une hiérarchisation fine s'impose : le choix s'effectue en privilégiant un niveau d'évaluation par rapport à un autre, et une tendance évaluative à l'intérieur de ce niveau. Les évolutions de points de vue suivent cette décision collective (Moscovici et Doise, 1992).

32 Au niveau général, les grandes valeurs semblent en contradiction entre elles. En situation intergroupe, elles s'activent, quel que soit le sujet de la discussion. La protection de la biodiversité (valeur intrinsèque ?) a tendance à régresser tandis que la protection de l'eau (survie humaine ?) reste stable. Cependant, dans les concertations 
observées, toutes deux sont abandonnées au cours de la décision au profit de valeurs purement sociales (absence de conflit, rencontre et concertation, reconnaissance sociale et identité) ${ }^{11}$, ce qui explique le peu d'efficacité sur le terrain. Dans les rencontres entre étudiants, comme dans les concertations, la valeur associée à la production agricole progresse ou se trouve reconnue au même niveau que la protection de l'eau (peut-être parce que, pour elle aussi, sa finalité ultime est la survie humaine ?). Chez les étudiants, les discussions sur les valeurs (D3) provoquent plus de mouvements d'opinion que celles sur les descriptifs de bordures (D2). Des conflits empêchent certains groupes de trouver un accord, mais c'est dans cette situation très impliquante que les étudiants en agriculture présentent une petite évolution dans le sens des bandes d'herbe le long des ruisseaux.

33 Le poids des lois et des règlements, qui institutionnalisent un certain nombre de valeurs clés pour chaque société, s'active dans la rencontre des différents groupes sociaux. Le droit de propriété (liberté individuelle) ou les règles imposées par les lois de protection de la nature (contrainte collective) apparaissent comme des socles incontournables et antagonistes, sorte de bases de repli pour chaque groupe, pour préserver ses intérêts propres.

Les normes sociales sont également agissantes. Si on y fait appel (D4), elles jouent leur rôle social régulateur et orientent les décisions collectives, tout en contraignant l'évolution des opinions. C'est ce que nous observons dans les groupes où une décision dichotomique est demandée, proposant, à l'aide de photos, une situation de compromis agri-environnemental versus une situation environnementale très dégradée. Dans ce cas, les accords se font massivement sur la norme nouvelle et les étudiants agricoles évoluent vers des positions prenant en compte la protection de l'eau. Les normes jouent également implicitement dans la dynamique de groupe, au cours de la discussion. C'est ce que nous observons dans le groupe agricole, dont l'histoire sociale et professionnelle a créé une forte cohésion. Les étudiants environnementaux présentent une consistance bien moins importante, socialement parlant, ce qui les fait abandonner leurs points de vue beaucoup plus facilement (y compris dans la situation normative D4). Dans les concertations observées, seule une association environnementale très militante (en $\mathrm{C}$ ) reste ferme sur ses positions : cela conduit à la réapparition du conflit.

Les intérêts socio-économiques sont au cœur du processus, dès lors que, sur le terrain, les changements attendus touchent les techniques, le travail et les coûts. La concertation devrait alors se transformer en négociation, où chacun pourrait défendre ses positions et prendre en compte celles des autres pour trouver un accord répartissant au mieux les avantages recherchés et les nouveaux coûts impliqués par le changement. En réalité, des mécanismes de pouvoir social entrent en jeu, de subtiles stratégies se développent pour ne pas aborder de front cette discussion épineuse. Les collectivités locales ne veulent pas payer les nouveaux services environnementaux demandés aux agriculteurs, qui ne veulent pas abandonner leurs libertés d'action sur leur territoire. Les concertations observées n'ont jamais abordé ces questions, et les opinions sur l'item "contrats» n'ont jamais progressé dans les rencontres entre étudiants.

Les désirs individuels, basés sur des sensations personnelles (beauté du paysage, bonheur à la campagne, amour de la nature) sont socialement partagées par tous (agriculteurs ou non) ${ }^{12}$. Ces valeurs « socio-environnementales» consensuelles, qui se 
rapportent à l'hédonisme ou à l'universalisme, permettent un climat convivial dans les discussions, mais ne suffisent pas à enclencher des changements réels, surtout quand les intérêts sociaux contradictoires ne sont pas négociés en tant que tels. C'est ce que nous constatons quand les membres d'une concertation s'accordent sur leur amour commun de la campagne ou quand les étudiants discutent de photos (D1). Les désaccords subsistent, les points de vue ne changent pas et on observe même un positionnement plus affirmé pour chaque groupe social sur le partage de l'espace (droit de propriété pour les étudiants agricoles, achat des terrains par les collectivités pour les étudiants environnementaux).

Ainsi, dans les rencontres, une sorte de confiance générale dans les valeurs exprimées au cours de la discussion a prévalu (convivialité, démocratie locale, reconnaissance mutuelle de la nécessaire protection de l'eau et de la production agricole). Cependant, au niveau du groupe social ou des individus, on observe peu de changements proenvironnementaux. Le conflit latent ayant le plus souvent été évité, les grandes structures organisationnelles et légales sont restées les repères des représentations et des actions concrètes. Étudiants et participants aux concertations ont généralement apprécié les rencontres, mais les points de vue n'ont pas évolué dans le sens attendu.

\section{Éthique et éducation à l'environnement : quelles idéologies?}

38 Les idéologies sont des systèmes de pensée qui s'auto-référencient (Deconchy, 1991). Ainsi, les valeurs et les représentations portées par les systèmes idéologiques, y compris éducatifs et scientifiques, paraissent souvent indépassables. La méthodologie employée (réalisme modeste, transdisciplinarité exigeante, choix d'un objectif précis) offre une base pour tenter de nous distancer de nos propres enfermements. Les deux études présentées ici soulèvent de nombreuses questions quant aux rôles des valeurs invoquées par l'éthique dans les choix individuels ou collectifs, mais également quant aux représentations de l'individu et des fonctionnements sociaux. L'éducation relative à l'environnement peut tirer profit de ces questionnements.

La société occidentale libérale est porteuse de valeurs particulières : liberté, autonomie, responsabilité, accomplissement individuel, hédonisme, etc. Les diverses structures d'éducation participent à la diffusion de ces valeurs, qui nous façonnent tous de manière implicite ou explicite. Imagine-t-on former des enfants irresponsables, sans projet individuel? Des adultes qui ne seraient pas autonomes? Les valeurs de solidarité, d'équité, de protection de l'environnement s'opposent à certains de ces principes ou n'y sont pas reliés. Comment le système éducatif peut-il traiter ces ambigüités? Jusqu'à quel point renforcer l'individualisme et l'attribution d'autoresponsabilité de l'enfant (Dubois, 1998) ? Est-ce compatible avec la recherche de finalités pro-sociales et pro-environnementales? À notre sens, dénier les conflits de valeurs ou réduire leur résolution à une responsabilité individuelle peut se révéler dangereux pour l'équilibre personnel des individus (Ehrenberg, 1998). Replacer ces valeurs dans leurs contextes historiques et idéologiques, les relier aux réalités sociales et humaines, aux lois, normes et contraintes physiques, permet de libérer chacun d'impératifs de choix démesurés. 

fonctionnements humains et sociaux (Beauvois, 1994). De récents modèles de psychologie et de neurosciences remettent en cause nos représentations d'un individu autonome et consistant. Les modèles duaux, expliquant d'un côté les habitudes et les stéréotypes, de l'autre les actions raisonnées, semblent particulièrement heuristiques (Smith et DeCoster, 2000). Ils dessinent les contours d'un être humain, certes moins glorieux que celui de nos visions humanistes, mais plus explicatif des réalités quotidiennes et historiques, parfois bien sombres. Quels enseignements en tirer pour l'éducation? Si nos conduites ne sont pas toujours dirigées par des raisonnements et des valeurs conscientes, quelle part faire aux autres types d'apprentissage? Quelle vision de lui-même proposons-nous à l'être que nous formons ? Éduquer aux contextes, aux contraintes et aux multiples déterminants de nos propres choix semble sans doute aussi important qu'éduquer à la liberté. Apprendre à repérer certains contextes sociaux, à les rechercher ou à les éviter, à les dénoncer ou à ne pas les mettre en place, etc. est peut-être plus efficace à long terme que d'interroger l'individu sur ses propres motivations.

41 La représentation des fonctionnements sociaux semble, elle aussi, en adéquation avec l'idéologie libérale. De même que les marchés économiques seraient la rencontre d'une offre et d'une demande atomisées et produiraient le juste prix, les choix sociopolitiques devraient être le produit de la libre discussion, de la transparence des informations et de la rencontre entre acteurs sociaux égaux. La démocratie et les concertations locales sont ainsi promues au rang de valeurs essentielles : qui oserait proposer le règne de l'arbitraire et du despotisme? Pourtant, ce raccourci dans la présentation des choix collectifs occulte l'ensemble des processus mis en évidence par les recherches: conflits, relations de pouvoir, lois et règlements, opacité et immobilisme des structures organisationnelles, réseaux sociaux, phénomènes intra et intergroupes, obéissance, influence, manipulation, etc. Quelles données fournit-on aux élèves et aux adultes en formation, concernant ces processus essentiels à leur compréhension du monde dans lequel ils sont plongés? Comment éduque-t-on aux choix collectifs?

Considérer notre rapport à l'environnement dans son aspect le plus concret nous fait prendre conscience de l'inévitable médiation sociale et technique des relations de chacun à la nature. Ainsi, mettre en œuvre des "valeurs environnementales ", qu'elles soient bio ou égo-centrées, passe obligatoirement par des références sociales et concrètes, par un "déjà-là » construit par la société et les générations passées. L'éducation relative à l'environnement n'échappe pas à la circularité reliant les finalités environnementales aux finalités sociales, elles-mêmes reflets de représentations du monde et de rapports de force historiques. Dans son objectif «former une personne citoyenne animée du désir d'action collective afin de construire le bien commun » (Ifrée, sans date), une sorte de pari sous-entend que le bien commun construit sera pro-environnemental, ou bien délègue à cette personne citoyenne une lourde responsabilité... Notre étude montre, comme beaucoup d'autres, que rien n'assure la concrétisation des valeurs environnementales dans les concertations. Au contraire, la complexité même des processus sociaux prédispose à la disparition des objectifs environnementaux. Quelle peut être la position de l'éducation relative à l'environnement face à ce constat? Pour notre part, il nous semble important, comme dans les recherches sur la complexité, de clarifier les objectifs de chaque formateur et 
de chaque action pédagogique. Dans chaque situation, la finalité éducative peut être différente. Développement personnel de l'individu? Amélioration des relations sociales? Amélioration de la situation environnementale? Ce type d'interrogation conduit à rechercher la précision dans ses objectifs et dans les moyens de les mettre en œuvre. Elle offre l'occasion de réfléchir aux conséquences idéologiques des choix pédagogiques. Il semble ainsi possible de construire une éducation inspirée par plusieurs valeurs contradictoires (individualistes, altruistes, environnementales...) pour en bâtir progressivement la synergie.

\section{BIBLIOGRAPHIE}

Ajzen, I. (2002). Residual effects of past on later behavior : habituation and reasoned action perspective. Personality and Social Psychology Review, 6, 107-122

Azjen, I. et Fishbein, M. (1980). Understanding attitudes and predicting social behavior. Englewood Cliffs (NJ) : Prentice Hall

Beauvois, J.-L. (1994). Traité de la servitude libérale. Paris : Dunod.

Billaud, J.-P. (1996). Négociations autour d'une nature muette. Études rurales, 141-142, 63-83. Boltansky, L. et Thevenot, L. (1991). De la justification : les économies de la grandeur. Paris : L'Harmattan.

Channouf, A., Py, J. et Somat, A. (1996). Prédire des comportements à partir des attitudes : nouvelles perspectives. In Deschamps, J.-C. et Beauvois, J.-L. (dir.), Des attitudes aux attributions (p. 55-65). Grenoble : Presses universitaires de Grenoble.

Deconchy, J.-P. (1991). Mécanismes idéologiques et représentations idéographiques. In Aebischer, V., Deconchy, J.-P. et Lipiansky, E.M. (dir.), Idéologies et représentations sociales (p. 117-127). Cousset : Delval.

Descola, P. (2005). Par delà nature et culture. Paris : Gallimard

Dewitte, J. (2001). Le déni du déjà-là : sur la posture constructiviste comme manifestation de l'esprit du temps. In Caillé, A., Chanial, P. et Vandenberghe, F. (dir.), Chassez le naturel... Écologisme, naturalisme, constructivisme (p. 393-409). Paris : La Découverte.

Dubois, N. (1998). Internalité et connaissance évaluative. In Beauvois, J.-L., Joule, R.-V. et Monteil, J.-M. (dir.), Vingt ans de psychologie sociale expérimentale francophone (p. 189-225). Grenoble : Presses Universitaires de Grenoble.

Ehrenberg, A. (1998). La fatigue d'être soi. Paris : Odile Jacob.

Feertchak, H. (1996). Les motivations et les valeurs en psycho-sociologie. Paris : Armand Colin. Georgescu-Roegen, N. (1979). Energy analysis and economic valuation. Southern Economic Journal, 45(4), 1023-1058.

Habermas, J. (1990). La technique et la science comme idéologie. Paris : Gallimard

Helkama, K. (1999). Recherches récentes sur les valeurs. In Beauvois, J.-L., Dubois, N. et Doise, W. (dir.), La construction sociale de la personne (p. 61-73). Grenoble : Presses Universitaires de Grenoble. 
Ifrée (sans date). Appel à communication du colloque de La Rochelle : Éthique et éducation à l'environnement. Consulté en mars 2009 sur : http://www.ifree.asso.fr.

Jollivet, M. (1992). Sciences de la nature, sciences de la société, les passeurs de frontières. Paris : CNRS Éditions.

Kergreis, S. (2001). Opinion publique et avis d'experts : regards croisés sur l'espace agricole en Ille et Vilaine. Document non publié à l'occasion du $25^{\mathrm{e}}$ Colloque de l'Association des Ruralistes Français (Tatihou, octobre 2001).

Kergreis, S. (2004). Régulations sociales et cognitives dans les concertations agri-environnementales. Thèse de Doctorat inédite, Université de Rennes 2.

Kergreis, S. (2007). Pratiques des agriculteurs sur leurs bordures de champ : une approche anthropologiste et régulationniste. In Gendron, C. et Vaillancourt, J.-G. (dir.), Environnement et sciences sociales : les défis de l'interdisciplinarité (p. 377-399). Québec : Presses de l'Université Laval.

Kergreis, S. et Thenail, C. (2008). Attitude et comportement des agriculteurs vis-à-vis de la gestion des bordures de champ en paysage de bocage. In Antoine, A. et Marguerie, D. (dir.), Bocages et Sociétés (p. 385-395). Rennes : Presses universitaires.

Lafaye, C. et Thévenot, L. (1993). Une justification écologique ? Conflits dans l'aménagement de la nature. Revue française de sociologie XXXIV, 495-524.

Legay, J.-M. (1997). L'expérience et le modèle : un discours de la méthode. Paris : INRA Éditions.

Mauss, M. (1936). Sociologie et anthropologie. Paris : Presses Universitaires de France (7e édition Quadrige, 1997).

Morin, E. (1997). Réforme de pensée, transdisciplinarité, réforme de l'université. Communication au congrès international « Quelle université pour demain? Vers une évolution transdisciplinaire de l'Université », Locarno, Suisse. Texte publié dans Motivation, 24.

Moscovici, S. et Doise, W. (1992). Dissension et consensus. Une théorie générale des décisions collectives. Paris : Presses Universitaires de France.

Paicheler, G. (1976). Norms and attitude change I : Polarization and styles of behavior. European Journal of Social Psychology, 6(4), 405-427. Passet, R. (1974). L'économique et le vivant. Paris : Payot.

Peeters, G. (1999). Valeur et évaluation. In Beauvois, J.-L., Dubois, N. et Doise, W. (dir.), La construction sociale de la personne (p. 247-257). Grenoble : Presses Universitaires.

Perugini, M. et Bagozzi, R.P. (2001). The role of desires and anticipated emotions in goal-directed behaviors : broadening and deepening the theory of planned behavior. British Journal of Social Psychology, 40, 79-98.

Schultz, P.W. et Zelezny, L. (1999). Values as predictors of environmental attitudes : evidence for consistency across 14 countries. Journal of Environmental Psychology, 19, 255-265.

Schwartz, S.H. (1992). Universals in the content and structure of values : theoretical advances and empirical tests in 20 countries. In Zanna, M.P. (dir.), Advances in experimental social psychology Volume 25 (p. 1-62). San Diego : Academic Press.

Schwartz, S.H. (2006). Les valeurs de base de la personne : théorie, mesures et applications. Revue française de sociologie, 47(4), 929-968.

Smith, E.R. et DeCoster, J. (2000). Dual-process models in social and cognitive psychology : conceptual integration and links to underlying memory systems. Personality and Social Psychology Review, 4(2), 108-131. 
Stern, P.C. et Dietz, T. (1994). The value basis of environmental concern. Journal of Social Issues, $50(3), 65-84$.

Theys, J. (2000). Les attentes en matière d'environnement et la demande d'expertise. Document non publié pour le Colloque « Environnement, expertise, science et société » (Paris, juin 2000).

\section{NOTES}

1. Voir Helkama (1999) pour une présentation des critiques et discussions du modèle de Schwartz.

2. Écologue du paysage et agronome du laboratoire de l'INRA SAD (Institut national de la recherche agronomique, Sciences pour l'Action et le Développement) Paysage, Rennes.

3. Laboratoires LARES et CRP2C-LAUREPS, Université de Rennes 2.

4. Le choix de l'objectif a été en grande partie déterminé par le financement apporté par le programme de recherche 2000-2005 du Ministère de l'Écologie français, intitulé «Concertation, décision, environnement ».

5. Les agriculteurs ont été interrogés dans le cadre de plusieurs groupes professionnels, par le biais d'une collaboration avec les Chambres d'agriculture de Bretagne.

6. Chaque élément du modèle est résumé par ses initiales, inscrites dans la figure 2 : chaque élément correspond à une ou plusieurs phrases du questionnaire.

7. Les phrases en italique correspondent à certaines phrases proposées dans le questionnaire pour mesurer les degrés d'accord. Elles ont été retenues par l'analyse de régression comme étant les phrases le plus souvent reliées à l'intention comportementale (par le biais des accords ou désaccords mesurés sur des échelles de 1 à 7).

8. Les étudiants discutaient par groupe de quatre ou cinq. Une première étude a fait ressortir les résultats de concertation dans les classes (intra-groupe), puis une seconde a permis l'organisation de rencontres interclasses (inter-groupes). Ces derniers résultats concernent 237 étudiants, soient 55 groupes.

9. Dans deux concertations sur trois (A et $B$ ), les plantations le long des routes ne protègent pas spécifiquement la ressource en eau. Dans le troisième cas (C), les aspects concrets ont été abandonnés dans les décisions finales. Dans les trois cas, les Chambres d'agriculture ou les agriculteurs restent maîtres de la mise en œuvre.

10. Les degrés d'accord étaient enregistrés sur des échelles de 1 (pas d'accord) à 7 (tout à fait d'accord).

11. Dans le questionnaire utilisé avec les étudiants, les valeurs en jeu concernaient uniquement l'environnement, décliné de la «biodiversité » jusqu'au "bonheur à la campagne » et la production agricole (productiviste ou agri-environnementale). Les autres valeurs sociales n'étaient pas mesurées. Celles-ci apparaissent dans les discussions au cours des trois concertations observées, dans les textes produits et dans les interviews de leurs participants.

12. Selon une autre enquête de l'auteure (voir Kergreis, 2004). 


\section{RÉSUMÉS}

Cet article propose de relier l'éthique aux actions individuelles et collectives, en observant comment les grandes valeurs qui donnent sens à notre vie s'incarnent dans des résultats environnementaux concrets. Une approche transdisciplinaire, qui relie différentes formes d'évaluation à plusieurs niveaux d'analyse, permet de repérer comment les valeurs générales se trouvent filtrées par différents prismes relatifs aux contextes sociaux et environnementaux. Le jeu des habitudes, des techniques, des intérêts ou des contraintes physiques interfère avec celui des désirs, des normes ou des lois dans les décisions prises. Deux études illustrent cette approche, l'une concernant un choix individuel, l'autre un choix collectif. Les résultats obtenus montrent la difficulté de concrétiser des "valeurs environnementales». Les éléments théoriques et méthodologiques présentés peuvent nourrir la réflexion sur les objectifs et les pédagogies employées en éducation relative à l'environnement.

This article proposes connecting ethics with individual and collective actions, by observing how the values which give direction to our life are embodied in concrete environmental results. A transdisciplinary approach, which connects various forms of evaluation with several levels of analysis, illustrates how general values are filtered by various prisms relative to social and environmental contexts. Customs, techniques, interests or physical constraints interfere with desires, standards or laws in decision making. Two cases illustrate this approach, one concerning an individual choice, the other a collective choice. The results show the difficulty in turning «environmental values" into reality. The theoretical and methodological elements presented can contribute to the reflection on the objectives and the pedagogies used in environmental education.

\section{AUTEUR}

\section{SYLVIE KERGREIS}

Ingénieure du génie rural, des eaux et forêts, et docteure en sciences humaines et sociales (Université de Rennes 2), elle est actuellement chercheure associée au Laboratoire de psychologie sociale du Centre de recherche sur la perception, la cognition et la communication (LAUREPSCRPCC), Université Rennes 2. Elle a été enseignante en sciences économiques et sociales, a coordonné un projet de recherche en collaboration avec deux laboratoires (psychologie sociale et écologie) et a été chargée de mission en éducation à l'environnement et au développement durable à la Direction générale de l'enseignement et de la recherche au Ministère de l'Agriculture. 